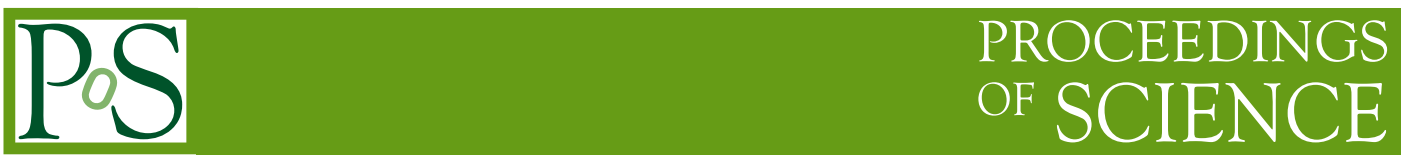

\title{
Scattering in Higher Dimensional Field Theories: Analyticity Properties and High Energy Behaviors
}

\author{
Jnanadeva Maharana* \\ Author affiliation \\ Institute of Physics, Bhubaneswar, India \\ Max-Planck Institute for Gravitational Physics, Albert Einstein Institute, Golm, Germany \\ E-mail: maharana@iopb.res.in
}

\begin{abstract}
The analyticity properties of scattering amplitude and its behavior at asymptotic energies in considered in the axiomatic LSZ formulation. The existence of Lehmann ellipse is proved. The analog of Froissart bound in $D$ spacetime dimensions is derived.
\end{abstract}

The 39th International Conference on High Energy Physics (ICHEP2018)

4-11 July, 2018

Seoul, Korea

*Speaker. 
The investigation of the properties of the theories in higher spacetime dimensions is of great importance. It is believed that the goal to achieve unification of the forces of Nature is to formulate theories which live in higher dimensions, $D>4$. The supersymmetric theories and supergravity theories exist in $D>4$. Moreover, the superstring theories which are consistently constructed perturbatively hold the prospect of unifying gravitational interaction with the standard model. However, in such theories the extra dimensions must be compactified so that one can describe physics at loweenergies i.e. energy scales accessible by present accelerators. It is argued that the compactification scale might be at the Planck scale or in the vicinity of the GUT scale. However, in certain alternative scenario, the compactification scales might be much lower. It is proposed that the decompactification might occur at LHC energy (see the two references for review for this proposal and the references therein [2,3]). Moreover, the limits on the large compactification scenario (large length scale) is not ruled of from the experiments at LHC. Thus there is considerable phenomenological research in the large radius compactification scenario. Therefore, it is worthwhile to investigate the analyticity properties of the scattering amplitude in higher dimensional field theories.

The content of this talk is a brief account of my investigations of the analyticity of scattering amplitude in higher dimensional $D>4$ field theories. The postulates of LSZ [1] are the starting point for a theory of massive neutral scalar field. We focus on study of the analyticity property of the scattering amplitude in the axiomatic formulation rather then appealing to any particular model. The analyticity properties of scattering amplitudes have been studied in detail for $D=4$ theories and we refer the readers to two important books for more details and further references $[4,5]$.

The axioms are: a1. The states of the system are represented in a Hilbert space, $\hat{\mathscr{H}}$. All the physical observables are self-adjoint operators in the Hilbert space, $\hat{\mathscr{H}}$.

a2. The theory is invariant under inhomogeneous Lorentz transformations.

a3. The energy-momentum of the states are defined. It follows from the requirements of Lorentz and translation invariance that we can construct a representation of the orthochronous Lorentz group. The representation corresponds to unitary operators, $\hat{U}(\hat{a}, \hat{\Lambda})$, and the theory is invariant under these transformations. Thus there are hermitian operators corresponding to spacetime translations, denoted as $\hat{P}_{\hat{\mu}}$, with $\hat{\mu}=0,1,2,3, D-1$ which have following properties:

$$
\left[\hat{P}_{\hat{\mu}}, \hat{P}_{\hat{v}}\right]=0
$$

If $\hat{\mathscr{F}}(\hat{x})$ is any Heisenberg operator then its commutator with $\hat{P}_{\hat{\mu}}$ is

$$
\left[\hat{P}_{\hat{\mu}}, \hat{\mathscr{F}}(\hat{x})\right]=i \hat{\partial}_{\hat{\mu}} \hat{\mathscr{F}}(\hat{x})
$$

It is assumed that $\hat{F}$ carries no explicit spacetime depedance. If one chooses a representation where the translation operators, $\hat{P}_{\hat{\mu}}$, are diagonal and the basis vectors $\mid \hat{p}, \hat{\alpha}>$ span the Hilbert space, $\hat{\mathscr{H}}$, such that

$$
\hat{P}_{\hat{\mu}}\left|\hat{p}, \hat{\alpha}>=\hat{p}_{\hat{\mu}}\right| \hat{p}, \hat{\alpha}>
$$

then more precise statements can be made:

- Existence of the vacuum: there is a unique invariant vacuum state $\mid 0>$ which has the property

$$
\hat{U}(\hat{a}, \hat{\Lambda})|0>=| 0>
$$


The vacuum is unique and is Poincaré invariant.

- The eigenvalue of $\hat{P}_{\hat{\mu}}, \hat{p}_{\hat{\mu}}$, is light-like, with $\hat{p}_{0}>0$. We are concerned only with massive stated in this discussion. If we implement infinitesimal Poincare transformation on the vacuum state then

$$
\hat{P}_{\hat{\mu}} \mid 0>=0, \text { and } \hat{M}_{\hat{\mu} \hat{v}} \mid 0>=0
$$

note that $\hat{M}_{\hat{\mu} \hat{v}}$ are the generators of Lorentz transformations.

a4. The locality: two bosonic local operators $\mathscr{O}_{1}(\hat{x})$ and $\mathscr{O}_{2}\left(\hat{x}^{\prime}\right)$ commute when they are separated by spacelike distance.

$$
\left[\hat{\mathscr{O}}_{1}(\hat{x}), \hat{\mathscr{O}}_{2}\left(\hat{x}^{\prime}\right)\right]=0,\left(\hat{x}-\hat{x}^{\prime}\right)^{2}<0
$$

Our Minkowski metric convention is: $\hat{x} . \hat{y}=\hat{x}^{0} \hat{y}^{0}-\hat{x}^{1} \hat{y}^{1}-\ldots-\hat{x}^{D-1} \hat{y}^{D-1}$. For a neutral scalar field, $\hat{\phi}(\hat{x}): \hat{\phi}(\hat{x})^{\dagger}=\hat{\phi}(\hat{x})$ i.e. $\hat{\phi}(\hat{x})$ is hermitian. $\hat{\Phi}$ transforms as a scalar under inhomogeneous Lorentz transformations

$$
\hat{U}(\hat{a}, \hat{\Lambda}) \hat{\phi}(\hat{x}) \hat{U}(\hat{a}, \hat{\Lambda})^{-1}=\hat{\phi}(\hat{\Lambda} \hat{x}+\hat{a})
$$

In the frameworks of general relativistic quantum field theories, for $D=4$,. the scattering amplitude, $F(s, t)$, is an analytic function of the center of mass energy squared, $s$, for fixed $t$, the momentum transfers squared. The dispersion relations in $s$ have been proved for $t$ is within LehmannMartin ellipse. This is derived from the axiomatic approach of LSZ [1]. Thus our task is to generalize these results to arbitrary $D>4$. Thus what are the steps neded to derive the dispersion relations and then prove the analog of Froissart bound on total cross sections for $D>4$ theories.?

First, to write a fixed-t dispersion relation, it is essential to prove the existence of Lehmann ellipses. The scattering amplitude, in $D>4$ admits a partial wave expansion; the basis functions being the Gegenbauer polynomials which converges in the domain $-1 \leq \cos \theta \leq+1$. It is required to find an enlarged domain of convergence for the amplitude, in $\cos \theta$ which is generalization of Lehmann's technique. In order to achieve this goal, we have to prove analog of Jost-Lehmann-Dyson theorem $[6,7]$ for $D>4$. Next, we need to study the crossing properties of the amplitude. We mention en passant, for $D=4$, the existence of the Lehmann ellipses does not lead to the Froissart bound. It is necessary to prove the Martin's theorem which is the enlargement of the domain of analyticity of the scattering amplitude. It is analytic the product of two complex domains: $D_{s} \otimes D_{t}$. Thus a generalized proof of Martin's theorem is required. In what follows, we shall summarize our results and we refer the reader to the two works which contains details $[8,9]$.

Let us incorporate the key features of the scattering amplitude, starting with the expression for the four point amplitude as derived in the LSZ framework. The scattering is described by

$$
\begin{aligned}
& <-p_{d}-p_{c} \text { out } \mid p_{a} p_{b} \text { in }>-<-p_{d}-p_{c} \text { in } \mid p_{a} p_{b} \text { in }> \\
& =-\frac{i}{(2 \pi)^{D-1}} \int d x^{D} d y^{D} e^{-i p_{c} \cdot x-i p_{b} \cdot y}\left(\square_{x}-m_{c}^{2}\right)\left(\square_{y}-m_{b}^{2}\right)<-p_{d}\left|R \phi_{c}(x) \phi_{b}^{\dagger}(y)\right| p_{a}> \\
& =-\frac{i}{(2 \pi)^{D-1}} \int d^{D} x d^{D} y e^{-i p_{c} \cdot x-i p_{d} \cdot y}\left(\square_{x}-m_{c}^{2}\right)\left(\square_{y}-m_{d}^{2}\right) \\
& <0\left|R \phi_{c}(x) \phi_{d}(y)\right| p_{a} p_{b} \text { in }>
\end{aligned}
$$

where $R \phi_{c}(x) \phi_{b}^{\dagger}(y)$ is the retarded product of fields and we have maintained the identities of fields to keep track of the fields reduced. We write $\phi_{b}^{\dagger}(y)$ for the same reason. Our convention is that 
$p_{a}+p_{b}+p_{c}+p_{d}=0$ and the Mandelstam variables are: $s=\left(p_{a}+p_{b}\right)^{2}, t=\left(p_{a}+p_{d}\right)^{2}, u=$ $\left(p_{a}+p_{c}\right)^{2}$ and $s+t+u=m_{a}^{2}+m_{b}^{2}+m_{c}^{2}+m_{d}^{2}=4 m^{2}$. The scattering amplitude is defined to be

$$
\begin{aligned}
& <-p_{d}-p_{c} \text { out } \mid p_{a} p_{b} \text { in }>-<-p_{d}-p_{c} \text { in } \mid p_{a} p_{b} \text { in }> \\
& =2 \pi \delta^{D}\left(p_{a}+p_{b}+p_{c}+p_{d}\right) F\left(p_{a}, p_{b}, p_{c}, p_{d}\right)
\end{aligned}
$$

We have used the relation $\left(\square_{x}-m_{c}^{2}\right)\left(\square_{y}-m_{d}^{2}\right)\left(R \phi_{c}(x) \phi_{d}(y)\right)=R\left(j_{c}(x) j_{d}(y)\right.$ with the tacit understanding that the r.h.s of the relation contains derivatives of $\delta$-functions in general. However, when Fourier transformed, they lead to polynomials in $s$ or t oru since the amplitude is Lorentz invariant. The presence of such terms do not affect the analyticity properties of the amplitude. Thus, the scattering amplitude (9) expressed as [?, ?]

$$
F\left(p_{a}, \ldots p_{d}\right)=-\int d^{D} z e^{i P \cdot z}<-p_{d}\left|R j_{c}\left(\frac{z}{z}\right) j_{b}^{\dagger}\left(-\frac{z}{2}\right)\right| p_{a}>
$$

where $P=\frac{\left(p_{b}-p_{c}\right)}{2}$. In deriving (10) we have reduced $c$ and $b$. If we reduce $c$ and $d$ the amplitude is expressed as

$$
F\left(p_{a}, \ldots p_{d}\right)=-\int d^{D} z e^{-i Q \cdot z}<0\left|R j_{c}\left(\frac{z}{2}\right) j_{d}\left(-\frac{z}{2}\right)\right| p_{a} p_{b} \text { in }>
$$

The two equations derived above for the amplitude, $F$, are special cases of a generic retarded function

$$
F_{R}(q)=\int d^{D} z e^{i q \cdot z} \theta\left(z_{0}\right)<Q_{f}\left|\left[j_{l}\left(\frac{z}{2}\right), j_{m}\left(-\frac{z}{2}\right)\right]\right| Q_{i}>
$$

$j_{l}$ and $j_{m}$ are two generic currents and indices take values $a, b, c, d$. The two states $\left|Q_{f}\right\rangle$ and $\left|Q_{i}\right\rangle$ carry D-dimensional momenta $Q_{f}$ and $Q_{i}$ respectively and these momenta are held fixed. Thus the argument of $F_{R}$ does not display $Q_{f}$ and $Q_{i}$ and we treat them as parameters for the discussions to follow. We define two more functions for our later conveniences.

$$
F_{A}=-\int d^{D} z e^{i q \cdot z} \theta\left(-z_{0}\right)<Q_{f}\left|\left[j_{l}\left(\frac{z}{2}\right), j_{m}\left(-\frac{z}{2}\right)\right]\right| Q_{i}>
$$

and

$$
F_{C}(q)=\int d^{D} z e^{i q \cdot z}<Q_{f}\left|\left[j_{l}\left(\frac{z}{2}\right), j_{m}\left(-\frac{z}{2}\right)\right]\right| Q_{i}>
$$

From above definitions, it follows that

$$
F_{C}(q)=F_{R}(q)-F_{A}(q)
$$

Since $F_{C}$ is commutator of two currents, we open up the commutator in terms of products of currents $j_{l}\left(\frac{z}{2}\right) j_{m}\left(-\frac{z}{2}\right)-j_{m}(-z / 2) j_{l}(z / 2)$. Then we introduce complete set of physical states, $\sum_{n}\left|p_{n} \alpha_{n}><p_{n} \alpha_{n}\right|=\mathbf{1}$ between the product of currents. This is how one obtains a spectral representation for $F_{C}(q)$ and then those for $F_{R}(q)$ and $F_{A}(q)$. The microcausality plays a very important role in deriving a representation for $F_{C}(q)$ and hence for the advanced and retarded functions.

For the $D>4$ theories, we have derived the generalized J-L-D theorem and shown that the singularities of $F_{R}(q)$ lie in the complex plane. This is the starting point to prove the existence of small and large Lehmann ellipses for our case. Moreover, we also prove, for the case of the four point amplitude, the crossing symmetry following the original arguments of Bros, Epstein and Glaser. 
Therefore, the results derived for the higher dimensional theories may listed as (i) the spectral representations for $F_{C}(q), F_{R}(q)$ and $F_{A}(q)$. (ii) The generalized J-L-D theorem and proof of the existence of the Lehmann ellipses and (iii) the crossing property of the four point amplitude. Therefore, fixed-t dispersion relations can be written down.

The next task is to identify the product domains of analyticity of the amplitude. We have to resort to the partial wave expansion. As an example consider $D=10$. The isometry group is $S O(9)$ for a massive scalar case. When we expand in the 'spherial harmonics', the amplitude depends on angular momenta denoted by $l_{2}, \ldots l_{5}$, magnetic quantum numbers $m_{1}, \ldots m_{4}$; the corresponding angles are $\theta_{2}, \ldots \theta_{5}$. The four azimuthal angles are $\phi_{1}, \ldots \phi_{4}$. The 'spherical harmonics' are

$$
Y_{m_{1} \ldots m_{4}}^{l_{2} . . l_{5}}\left(\theta_{2}, . . \theta_{5}: \phi_{1}, \ldots \phi_{4}\right)
$$

For the scattering of scalars, there is only one scattering angle since rest of the angles are integrated out. The basis functions are the Gegenbauer polynomials, $C_{l}^{\lambda}(\cos \theta)$. The partial wave expansion is [12]

$$
F^{\lambda}=A_{1} s^{-\lambda+1 / 2} \sum_{l=0}^{\infty}(l+\lambda) f_{l}^{\lambda} C_{l}^{\lambda}(\cos \theta)
$$

where $\lambda=\frac{1}{2}(D-3)$. The amplitude $F^{\lambda}(s, t)=F(s, t)$. We analysed the analyticity properties of $F^{\lambda}(s, t)$. The partial wave unitarity relation assumes the form

$$
0 \leq\left|f^{\lambda}\right|^{2} \leq \operatorname{Im} f^{\lambda} \leq 1
$$

Indeed, we derived the analog of Martin's theorem for the $D$-dimensional case utilizing partial wave unitarity and positivity properties of the absorptive amplitude and its $t$-derivatives. We showed that a domain, $|t|<R, R$ independent of $s$, where partial wave expansion converges and $R$ was determined in terms of $m$.

Therefore, (i) with the proof of J-L-D theorem, (ii) derivation of the Lehmann ellipses, (iii) crossing symmetry and (iv) demonstration of fixed- $t$ dispersion relation, generalized the proof the analyticity of the amplitude for $D>4$. We derived analog of the Froissart-Martin bound for $D>4$. In order to arrive at this bound we had proved the analog of Jin-Martin bound on the amplitude which fixed the number of subtractions required in writing the dispersion relation. The result is

$$
\left|F^{\lambda}(s, t=0)\right|<\text { Constant } s(\ln s)^{D-2}
$$

The desired bound is

$$
\sigma_{\text {total }} \leq B(\lambda)\left(\frac{1}{2 \sqrt{4 m^{2}-\varepsilon}}\right)^{D-2}(\ln s)^{D-2}
$$

where $B(\lambda)=\frac{2^{\lambda} \Gamma(\lambda) \Gamma(\lambda+1 / 2)}{\pi^{3 / 2} \Gamma^{2}(2 \lambda)}$.

Acknowledgements: A part of this work was carried out at the Albert Einstein Institute of Gravitational Physics, The Max-Planck Institute, Golm The gracious hospitality of Hermann Nicolai is gratefully acknowledged.

\section{References}

[1] H. Lehmann, K. Symanzik and W. Zimmermann, Nuovo Cimento, 1, 205 (1955) 
[2] A. Antoniadis and K. Beneki, Mod. Phys. Lett. A 30, 1502002 (2015) for a recent review.

[3] D. Luest and T. R. Taylor, Mod. Phys. Lett. A 30, 15040015 (2015) for a recent review.

[4] A. Martin, Scattering Theory: unitarity, analyticity and crossing, Springer-Verlag, Berlin-Heidelberg-New York, (1969).

[5] A. Martin and F. Cheung, Analyticity properties and bounds of the scattering amplitudes, Gordon and Breach, New York (1970).

[6] R. Jost and H. Lehmann, Nuovo Cimen. 5, 1598 (1957).

[7] F. J. Dyson, Phys. Rev. 110, 1460 (1958).

[8] J. Maharana, J. Math. Phys. 58, 012302 (2017).

[9] J. Maharana, Phys. Lett. B 764, 212 (2017).

[10] H. Lehmann, Nuovo Cimen. 10, 579(1958).

[11] A. Martin, Nuovo. Cimen. 42, 930 (1966).

[12] Soldate, Phys. Lett. B 197], 321 (19870. 